\title{
Promove-Universitários: Efeitos na promoção de interações sociais e saúde mental
}

\author{
Laisa Aparecida Moretto \\ Alessandra Turini Bolsoni-Silva
}

\begin{abstract}
RESUMO
Para satisfatório desempenho na universidade, é relevante um repertório de habilidades sociais desenvolvido, o qual nem sempre está presente, o que pode trazer implicações para a saúde mental. O presente estudo teve como objetivo avaliar os efeitos do PromoveUniversitários, em estudantes de primeiro e segundo anos, valendo-se de um delineamento experimental de grupo, com medidas repetidas. Participaram catorze universitários, nove no Grupo Experimental e cinco no Controle, distribuídos aleatoriamente, os quais eram equivalentes nas medidas iniciais. Foram aplicados instrumentos para avaliar habilidades sociais e saúde mental, cujos dados foram analisados estatisticamente nas comparações entre os grupos. Os resultados demonstraram aquisições comportamentais, estatisticamente significativas, no Grupo Experimental para habilidades sociais e saúde mental, que se mantiveram nas medidas de seguimento. A mesma análise entre as linhas de base 1 e 2 do Grupo Controle não indicou mudança estatisticamente significativa. Considerando os resultados, conclui-se que o Promove-Universitários se mostrou efetivo ao proposto.
\end{abstract}

Palavras-chave: terapia comportamental; treinamento de habilidades sociais; universitários; delineamento experimental

\section{ABSTRACT}

\section{Promoting-Academics Program: Effects on the promotion of social interactions and mental health}

It is needed a developed repertoire of social skills in order to achieve a satisfying development in the university, although it is not always present, which can bring consequences to mental health. This study aimed to assess the effects of the Promoting-Academics Program in freshmen and sophomores through a group experimental design, with repeated measures. Fourteen students participated in the study - nine in the Experimental Group, five in the Control Group - who were randomly distributed and presented equivalent initial measures. Instruments were applied to assess social skills and mental health whose data were statistically analyzed in comparisons between the groups. Results showed statistically significant behavior acquisitions in the Experimental Group for social skills and mental health, which were maintained in the follow-up measures. The same analysis between 1 and 2 baselines in the control group did not indicated any statistically significant chance. Considering the results, the conclusion is that the Promoting-Academics Program revealed itself effective.

Keywords: behavior therapy; social skills training; college students; experimental design.

A população universitária no Brasil vem crescendo progressivamente nos últimos anos, mas, por outro lado, apenas um terço dela concluiu o curso (Instituto Nacional de Pesquisas -[INEP], 2014). Ainda que o INEP (2014) não aponte para possíveis razões desses números, o estudo de Brandão (2016) constatou que problemas de saúde mental e déficits de habilidades sociais, no ingresso e no segundo ano da universidade, predisseram desempenho acadêmico inicial insuficiente, o qual, por sua vez, influenciou tanto o desempenho acadêmico final

\section{Sobre os Autores}

L. A. M.

orcid.org/0000-0001-9459-7116

Universidade Estadual Paulista

(Unesp) - Bauru, SP

laisamoretto@hotmail.com

A. T. B.

orcid.org/0000-0001-9459-7116 Universidade Estadual Paulista

(Unesp) - Bauru, SP

bolsoni.silva@unesp.br

\section{Direitos Autorais}

Este é um artigo de acesso aberto e pode ser reproduzido livremente, distribuído,

transmitido ou modificado, por qualquer pessoa desde que usado sem fins comerciais. 0 trabalho é disponibilizado sob a licença Creative Commons CCBY-NC.

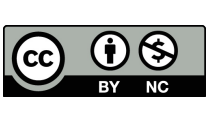


quanto a conclusão do curso (Brandão, Bolsoni-Silva, \& Loureiro, 2017). Na mesma direção, Tang, Byrne, e Qin (2018) identificaram que indicadores de problemas de saúde mental aumentavam o risco de comportamento suicida.

Desse modo, torna-se relevante identificar habilidades sociais para o contexto universitário, como as habilidades de comunicação, enfrentamento e afeto (Leme, Del Prette, \& Del Prette, 2016). Tais classes de comportamentos são relevantes para as demandas na vida universitária, as quais implicam em interações sociais com colegas/amigos, autoridades, namorados, familiares (Bolsoni-Silva \& Loureiro, 2017; Ribeiro \& Bolsoni-Silva, 2011). Cabe destacar que o estudar/fazer trabalho sozinho não implica necessariamente em comportamento social e, sim, em organização de rotina e tarefas, autoconhecimento e autocontrole (Skinner, 1974) o que também precisa ser considerado em intervenções com essa população.

0 termo habilidades sociais (HS) remete a comportamentos operantes (A. Del Prette \& Del Prette, 2001; Z. A. P. Del Prette \& Del Prette, 2008), mantidos pelos efeitos que causam no ambiente e que visam aumentar os ganhos e diminuir as perdas para os envolvidos na interação social (Bolsoni-Silva, 2002; Bolsoni-Silva \& Carrara, 2010), podendo ser considerados como funcionalmente equivalentes para problemas de saúde mental (Goldiamond, 2002), uma vez que um amplo repertório facilita o enfrentamento de eventos estressantes (Segrin \& Flora, 2000). Treinamento em habilidades sociais podem prevenir problemas em saúde mental da população em geral (Abreu, Miranda, \& Murta, 2016).

Estudos de intervenção tendo por foco o treino em comunicação (Klein et al., 2011) atestaram a melhoria da saúde mental dos participantes. Klein et al. (2011) demonstraram, em um delineamento experimental, que o ensino de resolução de problemas interpessoais com 491 pessoas com depressão crônica diminuiu os sintomas depressivos, mas não foi realizado com universitários. Na mesma direção, Beidel et al. (2014) aplicaram treinamento de habilidades sociais, em delineamento experimental, com 106 pacientes adultos que apresentavam ansiedade social. Em um dos grupos usou-se a terapia de exposição (imaginação ou ao vivo) e no outro grupo de intervenção, além da terapia de exposição, também incluiu treinamento de habilidades sociais. Os resultados mostraram que ambos os grupos tiveram mais ganhos que o Grupo Controle, mas o grupo que incluiu o treinamento de habilidades sociais apresentou menores indicadores de depressão crônica e maior ocorrência de habilidades sociais.

Propriamente com universitários encontram-se estudos com treinamento em habilidades sociais (e. g., Probst, Geib,
Güroff, \& Mühlberger, 2017) e intervenções que fizeram uso de terapia comportamental (e. g., Räsänen, Lappalainen, Muotka, Tolvanen, \& Lappalainen, 2016). No estudo de Probst et al. (2017), 40 estudantes foram distribuídos aleatoriamente em dois grupos de intervenção, em um dos grupos foi ensinado o dizer não e em outro foi ensinado o comportamento de pedir/exigir; o estudo demonstrou que cada grupo foi capaz de aprender a habilidade social treinada diretamente, mas não a outra. Räsänen et al. (2017) distribuíram aleatoriamente 68 universitários em um grupo de intervenção em terapia de aceitação e compromisso aplicada online, em sete semanas, e um Grupo Controle; o grupo intervenção foi reavaliado após 12 meses. O grupo de intervenção teve significativamente mais relato de bem-estar, satisfação de vida e reduziram sintomas de ansiedade e de depressão, que foram mantidos na avaliação de seguimento.

A partir dessa revisão, nota-se a relevância do ensino de comportamento social para pessoas com ansiedade ou depressão, bem como o uso do treinamento de habilidades sociais ou da terapia comportamental para melhorar os indicadores sociais e de saúde de universitários. No entanto, ainda é escassa a avaliação da terapia comportamental que faz uso do treinamento em habilidades sociais com foco na análise funcional e na resolução de problemas, incluindo, portanto, o ensino de múltiplos comportamentos sociais valendo-se de procedimentos diversos.

Em revisão da literatura nacional com intervenções com universitários na interface com habilidades sociais e indicadores de saúde mental verificou-se que: a) as intervenções são semanais; b) variando em número entre 10 (Ferreira, Oliveira, \& Vandenberghe, 2014), 12 (Orti, SouzaGirotti, \& Bolsoni-Silva, 2015; Lima \& Soares, 2015; Rocha, Bolsoni-Silva, \& Verdu, 2012), (Lopes, Dascanio, Ferreria, Del Prette, \& Del Prette, 2017) ou 20 sessões (Bolsoni-Silva, Leme, Lima, Costa-Júnior, \& Correia, 2009); e c) o número de participantes variou de 3 (Orti et al, 2015; Rocha, et al., 2012), 11 (Lima \& Soares, 2015), a 35 pessoas (Lopes et al., 2017). Quanto às técnicas utilizadas, Lopes et al. (2017) descreveram o uso de técnicas comportamentais, Ferreira et al. (2014) indicaram técnicas cognitivas e comportamentais e os estudos de Bolsoni-Silva et al. (2009), Orti et al. (2015) e de Rocha et al. (2012) circunscrevem o uso da terapia analítico-comportamental. Quanto às medidas de saúde mental de ansiedade e depressão, os estudos de Ferreira et al. (2014), de Orti et al. (2015) e de Rocha et al. (2012) apontaram para a diminuição dos sintomas. A avaliação de seguimento foi conduzida após três (Lopes et al., 2017) e seis meses (Ferreira et al., 2014; Orti et al., 2015; Rocha et al., 2012).

O estudo de Lopes et al. (2017) aplicou um programa de 


\section{H. INTERACÃO EM LF PSICOLOGIA}

treinamento em habilidades sociais com foco no desenvolvimento interpessoal profissional em universitários de exatas e, após a intervenção, os estudantes tiveram ganhos significativos. Todos os estudos nacionais mencionados relataram melhora, ou na saúde mental ou na aquisição de repertório de habilidades sociais. Entretanto, quanto a delineamento, nenhum deles foi conduzido em delineamento experimental de grupo.

Intervenções direcionadas à promoção de habilidades sociais tendo por foco a terapia comportamental em universitários são também escassas na literatura nacional. A Terapia Analítico-Comportamental (TAC), para Meyer et al. (2010), é “... uma forma de prestação de serviços que utiliza o arcabouço teórico da análise do comportamento e o conhecimento de pesquisas básicas e aplicadas para a solução de problemas humanos" (p. 172) e afirma-se que ela tem por principal instrumento de avaliação e de intervenção a análise funcional (Abreu \& Abreu, 2017).

0 estudo de Bolsoni-Silva et al. (2009) descreveu resultados de um programa nacional nomeado como Programa Universitários que possui dados de eficácia, em um delineamento quase-experimental com pré e pós-teste. Esse programa foi desenvolvido numa universidade pública em 2002. Trata-se de uma intervenção analítico-comportamental que se iniciou tendo como foco a promoção de habilidades sociais e outros comportamentos na resolução de problemas relacionados à vida universitária, sendo vinculada a Estágio Supervisionado em Terapia Comportamental, contendo 20 sessões (Bolsoni-Silva et al., 2009). Em um segundo momento o programa foi testado, em delineamento de sujeito único, em um formato com 12 sessões, no atendimento individual com universitários que apresentavam fobia social (Rocha et al., 2012) e/ou depressão (Orti et al., 2015).

Além da relevância científica da presente pesquisa, ela também tem relevância social considerando a promoção de saúde e prevenção de problemas de saúde mental que podem levar a evasão e ao suicídio, podendo contribuir com a política pública. Este estudo objetivou avaliar os efeitos do Promove-Universitários, em um desenho experimental de grupo, considerando medidas de habilidades sociais e de saúde mental (ansiedade e depressão).

\section{MÉTODO}

\section{DELINEAMENTO}

O delineamento experimental (Cozby, 2003) foi elaborado como descrito a seguir: os participantes foram divididos aleatoriamente em Grupo Experimental e Grupo Controle. Em seguida o Grupo Experimental passou pela intervenção de três meses, enquanto o Controle ficou em espera. Ao término da aplicação do Promove-Universitários, todos foram novamente avaliados. Após seis meses do término da intervenção, apenas o Grupo Experimental foi novamente avaliado no seguimento. Por questões éticas, após a intervenção com o Grupo Experimental, o Grupo Controle também passou por intervenção. Nesse delineamento, a variável independente é a intervenção, enquanto que as variáveis dependentes são as medidas de habilidades sociais, depressão e ansiedade.

\section{PARTICIPANTES}

Participaram 14 graduandos, separados aleatoriamente em condição experimental $(n=9)$ e em condição controle $(n=5)$. Sete deles matriculados em uma universidade pública e sete em uma faculdade particular. Todos estavam, obrigatoriamente, entre o primeiro e o segundo anos durante o período da intervenção e tinham idade média de 22,64 anos $(\mathrm{DP}=8,50)$.

\section{INSTRUMENTOS}

Inventário de Habilidades Sociais - IHS-Del-Prette: instrumento de autorrelato, desenvolvido por Z. A. P. Del Prette \& Del Prette (2001), com a finalidade de avaliar respostas comportamentais diante de situações de enfrentamento social. 0 inventário conta com 38 itens, cada um descreve uma relação interpessoal e uma possível reação, que estão agrupados em cinco fatores. Estudos psicométricos indicaram índices de discriminação positivos; com um alfa de Cronbach de 0,75; e estrutura fatorial com cinco fatores principais (F1 - Autoafirmação e enfrentamento com risco; F2 - Autoafirmação na expressão de sentimento positivo; F3 - Conversação e desenvoltura social; F4 Autoexposição a desconhecidos e situações novas; F5 Autocontrole da agressividade), identificados em termos comportamentais-situacionais, que explicaram 92,75\% da variância total (Z. A. P. Del Prette \& Del Prette, 2001).

Inventário de Fobia Social (Mini-Spin): versão abreviada do Inventário de Fobia Social (SPIN - Connor, Kobak, Churchill, Katzelnick, \& Davidson, 2001) possibilita o diagnóstico do Transtorno de Ansiedade Social (TAS). É composto por três itens: 1) Evito fazer coisas ou falar com certas pessoas por medo de ficar envergonhado; 2) Evito atividades nas quais sou o centro das atenções; e 3) Ficar envergonhado ou parecer bobo são meus maiores temores. Esses itens são avaliados em uma escala tipo Likert que varia de 0 a 4 (nada, um pouco, moderadamente, bastante, extremamente). 0 instrumento apresentou boas propriedades psicométricas: 


\section{W INERACÄOEM ET PSICOLOGIA}

$89,8 \%$ de precisão no diagnóstico do TAS, especificidade de $90 \%$, sensibilidade de $88,7 \%$, valor preditivo positivo de $52,6 \%$ e valor preditivo negativo de $98,5 \%$ (Osório, Crippa, \& Loureiro, 2007).

Inventários de Ansiedade e de Depressão Beck - BAl e BDI: essas escalas foram elaboradas por Beck e colaboradores (Beck \& Steer, 1993; Beck, Epstein, Brown, \& Steer, 1998) e validadas, no Brasil, por Cunha (2001). As escalas são constituídas por 21 itens, avaliados pelo indivíduo numa escala Likert de 4 pontos. As características psicométricas se mostraram satisfatórias para a população universitária: com alfa de Cronbach de $0,87, \mathrm{M}=7,54, \mathrm{DP}=6,88$ para o BAl; e alfa de Cronbach $(0,82), M=6,47, D P=5,80$ para o BDI (Cunha, 2001). Informa-se que no período da pesquisa as escalas Beck utilizadas ainda constavam como de uso favorável no sistema SATEPSI, que foi desenvolvido pela Gerência de Tecnologia da Informação do Conselho Federal de Psicologia (CFP), com o objetivo de regular a utilização de testes psicológicos.

Questionário de Avaliação de Habilidades Sociais, Comportamentos e Contextos para Universitários - QHCUniversitários: instrumento desenvolvido e validado por Bolsoni-Silva e Loureiro (2017) é composto por questões referentes à maneira e frequência com que os alunos se comportam em relação a seus pais, amigos, namorado(a), companheiros de república e público desconhecido. 0 instrumento é organizado em duas partes: Parte 1 que contém 19 questões de frequência, avaliando três fatores: a) Comunicação e Afeto; b) Enfrentamento; e c) Falar em Público. Já a Parte 2 é composta por 217 opções de respostas, que compõe dois fatores (potencialidades e dificuldades) que incluem a avaliação quanto à qualidade de interações sociais em diversos contextos, incluindo pais, irmãos, colegas, amigos e namorados. Tanto a Parte 1 quanto a Parte 2 possuem características psicométricas satisfatórias: com alfa Cronbach de 0,657 para ambas as partes do instrumento, com variância de 66,27 para a Parte 1 e variância de 71,90 para a Parte 2. As Partes 1 e 2 possuem validade discriminante quanto a indicadores de ansiedade e depressão e validade concorrente com o IHS-Del Prette.

Roteiro de entrevista semiestruturada: o instrumento está descrito em Bolsoni-Silva et al. (2009), que apresenta questões-guias para obter rapport e descrever funcionalmente queixas ou dificuldades, o qual subsidia a formulação do caso e os objetivos individuais de atendimento. As queixas dos participantes são colhidas a partir desse instrumento.

Roteiro de avaliação de satisfação de sessão: elaborado pelas autoras deste texto, tem o objetivo de colher informações sobre a experiência e avaliação do atendimento
Laisa Aparecida Moretto e Alessandra Turini Bolsoni-Silva

por parte do universitário no que se refere ao tema do encontro, à condução pelo terapeuta e quanto à própria participação. $O$ instrumento foi composto por um quadro para que fosse indicado: 1. Satisfatório; 2. Parcialmente satisfatório; e 3. Uma sugestão para as questões.

\section{CARACTERISTIICAS DO PROGRAMA PROMOVE- UNIVERSITÁRIOS}

Foi aplicada no presente estudo a intervenção descrita por Bolsoni-Silva (2009b) e avaliada por Bolsoni-Silva et al. (2009), Rocha et al. (2012) e por Orti et al. (2015). Cada uma das doze sessões, que ocorreram uma vez por semana com duração de duas horas, foram conduzidas pela primeira autora. O programa prevê o uso de uma cartilha informativa como orientação ao terapeuta e como biblioterapia para o cliente (Bolsoni-Silva, 2009a). Os comportamentos de habilidades sociais ensinados envolvem comunicação, afeto e enfrentamento, e foram selecionados considerando o campo teórico prático das habilidades sociais (A. Del Prette \& Del Prette, 2001) e um estudo de caracterização de demandas de universitários que buscavam por atendimento psicológico (Ribeiro \& Bolsoni-Silva, 2011), que destacou a necessidade do ensino de habilidades sociais contingentes às interações sociais com amigos, colegas, ambiente de república, lidar com autoridade, falar em público e relacionamento amoroso; adicionalmente comportamentos de lidar com tarefas e trabalhar sozinho, que são queixas relacionadas com ansiedade, foram também trabalhados no programa. Bolsoni-Silva e Loureiro (2017) confirmaram empiricamente que tais comportamentos diferenciavam estudantes com e sem problemas de saúde mental.

É preciso estar previsto, adicionalmente, o ensino de outros comportamentos que envolve a busca por reforçadores (Ferster, 1973), como ler um livro, ver um filme, cozinhar, exercitar-se, dentre outros. O mesmo raciocínio ocorre nos casos de ansiedade social, em que a exposição a situações ansiogênicas (Zamignani \& Banaco, 2005) são importantes e, portanto, estimuladas. Em casos em que há problemas com organizar o estudo, foi necessário orientar o planejamento da rotina diária, semanal e mensal, bem como monitorar e reforçar a condução do planejamento.

Desse modo, as avaliações iniciais, que ocorreram individualmente, foram utilizadas para a formulação dos casos de forma a entender as queixas/dificuldades de maneira funcional e identificar quais comportamentos deveriam ser foco, em cada sessão, considerando individualmente os participantes. Os procedimentos utilizados são da terapia comportamental, como discussão em grupo da tarefa de casa (observação e registro semanal), feedback por escrito das tarefas entregues, modelagem, 


\section{H. INTERACÃO EM LF PSICOLOGIA}

modelação, role-playing, exercícios (A. Del Prette \& Del Prette, 2001), biblioterapia e principalmente perguntas de reflexão com o objetivo de promover autoconhecimento, descrevendo funcionalmente os comportamentos problemas e identificando comportamentos alternativos que incluem as habilidades sociais ensinadas. O terapeuta pode recorrer à cartilha para destacar um ou outro comportamento como modelo para a busca de soluções interpessoais, entretanto o foco na sessão não é a apresentação da cartilha. Toda sessão, com exceção da primeira, é iniciada com discussão da tarefa de casa, em que os problemas e as aquisições são analisadas funcionalmente e consequenciadas, momento também em que o grupo é estimulado a avaliar e encontrar soluções para as dificuldades relatadas.

Em um segundo momento, o comportamento-alvo daquele encontro é discutido a partir de perguntas abertas do tipo "o que é comunicação para vocês? ... quando vocês acham fácil conversar? ... quando é difícil? ... com quem? ... por que vocês acham que isso acontece? ... vocês pensam em como poderia ser diferente? .... de que forma vocês acham que esse comportamento poderia ajudar a resolver os problemas que vocês encontram no dia a dia?". A terceira parte da sessão tem por foco o treino de repertório que pode ser a partir de vídeos, exercícios ou role-playings, de preferência de situações relatadas pelos clientes. 0 último momento do encontro é destinado à avaliação e a entrega da tarefa de casa individuais e/ou coletivas.

\section{COLETA DE DADOS}

A primeira providência, após a aprovação pelo Comitê de Ética em Pesquisa (CAEE no 16475913.8.0000.5398, Parecer $n^{\circ}$ 323.436) foi a divulgação para os universitários dos primeiros e segundos anos de uma universidade pública e de uma faculdade particular. Após a divulgação, 72 alunos, considerando ambos os campi, contataram a pesquisadora para o atendimento; destes, ao receberem mais informações sobre o atendimento, 32 aceitaram participar da pesquisa e compareceram na avaliação inicial. Foi realizado sorteio usando-se papéis, de modo que 16 compuseram o Grupo Experimental (GE) e 16 o Grupo Controle (GC). No entanto, seis participantes do Grupo Experimental e 11 do Grupo Controle abandonaram o estudo antes do início da intervenção. Os dados foram colhidos por meio da aplicação dos instrumentos descritos, em sessões individuais, com média de 50 minutos cada.

\section{TRATAMENTO DE DADOS}

Os instrumentos foram tabulados conforme instruções próprias. Os dados foram organizados separando os grupos
Experimental e Controle. A primeira análise refere-se ao software Psicoinfo (Z. A. P. Del Prette \& Del Prette, 2008) que utiliza o método JT (Jacobson \& Truax, 1991, citado por Z. A. P. Del Prette \& Del Prette, 2008) para avaliar a significância clínica e o índice de mudança confiável da intervenção realizada. A segunda corresponde ao tratamento estatístico: a) o teste de Mann-Whitney usado para comparar duas amostras independentes (Grupo Controle e Experimental) para todas as medidas de interesse do estudo (habilidades sociais, ansiedade e depressão) nas medidas iniciais, para verificar se as amostras eram equivalentes; e b) o teste de Wilcoxon, para comparar duas amostras pareadas no Grupo Experimental e Controle. Foram consideradas significâncias a $5 \%$.

\section{RESULTADOS}

Nesta seção são apresentados os resultados de forma a comparar os grupos Experimental e Controle quanto às variáveis de interesse. Antes de comparar os grupos de forma a avaliar os efeitos da intervenção, foram conduzidas análises de Mann-Whitney quanto às medidas iniciais no que se refere às habilidades sociais, ansiedade e depressão, cujos resultados revelaram que os Grupos Controle e Experimental eram equivalentes estatisticamente quanto às medidas iniciais. A Tabela 1 apresenta as queixas dos participantes, bem como a frequência de ocorrência em ambos os grupos, nas medidas de avaliação.

Tabela 1. Levantamento e frequência das queixas dos participantes, em escores brutos

\begin{tabular}{|c|c|c|c|c|}
\hline \multicolumn{5}{|c|}{ Levantamento de queixas dos participantes $(n=14)$} \\
\hline \multirow{2}{*}{ Queixa inicial } & \multicolumn{2}{|c|}{ Experimental $(n=9)$} & \multicolumn{2}{|c|}{ Controle $(n=5)$} \\
\hline & Pré-teste & Pós-teste & $\begin{array}{l}\text { Linha } \\
\text { base } 1\end{array}$ & $\begin{array}{l}\text { Linha base } \\
2\end{array}$ \\
\hline $\begin{array}{l}\text { Dificuldade de falar em } \\
\text { público }\end{array}$ & 9 & 2 & 5 & 4 \\
\hline $\begin{array}{l}\text { Dificuldade em } \\
\text { relacionamento } \\
\text { interpessoal }\end{array}$ & 9 & 3 & 4 & 5 \\
\hline $\begin{array}{l}\text { Dificuldade em manter } \\
\text { relacionamentos mais } \\
\text { íntimos }\end{array}$ & 6 & 3 & 4 & 4 \\
\hline $\begin{array}{l}\text { Dificuldade em } \\
\text { relacionamento familiar }\end{array}$ & 7 & 2 & 3 & 3 \\
\hline $\begin{array}{l}\text { Dificuldade em manter } \\
\text { vínculos de amizade }\end{array}$ & 7 & 1 & 5 & 5 \\
\hline $\begin{array}{l}\text { Dificuldade em controlar } \\
\text { agressividade }\end{array}$ & 6 & 0 & 3 & 4 \\
\hline $\begin{array}{l}\text { Dificuldade em entender } \\
\text { demandas das relações } \\
\text { interpessoais }\end{array}$ & 7 & 1 & 3 & 3 \\
\hline
\end{tabular}




\section{W INERACĀOEM IT PSICOLOGIA}

De acordo com a Tabela 1, nota-se que a maioria das queixas dos universitários se relacionavam com a exposição em público, que incluía a dificuldade em apresentar seminário, dar recados para a sala, falar para um grupo de amigos ou em alguma situação que sua presença ficasse em evidência. As demais queixas referiam-se ao relacionamento interpessoal de amizade, familiar e íntimo. Dificuldade em entender demandas das relações interpessoais envolve déficit de autoconhecimento, o que foi superado em 6 de 7 participantes do GE. Após a intervenção, no Grupo Experimental, verificou-se uma redução, para a maioria dos participantes, do relato de queixas em todas as categorias investigadas, o que não foi observado no Grupo Controle. No que se refere à satisfação com a intervenção, colhida pelo Roteiro de avaliação de satisfação de sessão, a grande maioria das sessões do procedimento foram consideradas satisfatórias pelos participantes, em que apenas dois participantes consideraram como parcialmente satisfatória uma das sessões.

A Tabela 2 sumariza os achados nas diferentes avaliações quanto aos instrumentos de saúde mental e habilidades sociais na comparação dos grupos.

Tabela 2. Descrição, em escores brutos e porcentagem, das medidas de saúde mental e habilidades sociais para os grupos Experimental e Controle

\begin{tabular}{|c|c|c|c|c|c|c|c|c|c|c|c|}
\hline \multirow{4}{*}{ Indicadores } & \multicolumn{5}{|c|}{$\begin{array}{l}\text { Participantes clínicos do Grupo } \\
\text { Experimental e Grupo Controle }\end{array}$} & \multicolumn{6}{|c|}{$\begin{array}{l}\text { Índice de Significância Clínica } \\
\text { (ISC) dos fatores dos instrumentos }\end{array}$} \\
\hline & \multirow{2}{*}{\multicolumn{3}{|c|}{$\begin{array}{c}\begin{array}{c}\text { Grupo } \\
\text { Experimental }\end{array} \\
(\mathrm{N}=9)(\%)\end{array}$}} & \multirow{2}{*}{\multicolumn{2}{|c|}{$\begin{array}{c}\text { Grupo } \\
\text { Controle } \\
(\mathrm{N}=5)(\%)\end{array}$}} & \multirow{2}{*}{\multicolumn{3}{|c|}{$\begin{array}{c}\begin{array}{c}\text { Experimental } \\
(n=9)\end{array} \\
\text { Pré/Pós }\end{array}$}} & \multicolumn{3}{|c|}{ Controle $(n=5)$} \\
\hline & & & & & & & & & & B1/L & \\
\hline & Pré & Pós & Seg & LB1 & LB2 & PF & MF & MD & $\mathrm{PF}$ & MF & MD \\
\hline QHC - Fator I/Ansiedade & $\begin{array}{c}7 \\
(78)\end{array}$ & $\begin{array}{c}7 \\
(78)\end{array}$ & $\begin{array}{c}5 \\
(56)\end{array}$ & $\begin{array}{c}3 \\
(60)\end{array}$ & $\begin{array}{c}5 \\
(100)\end{array}$ & 2 & 0 & 0 & 0 & 0 & 0 \\
\hline QHC - Fator I/Depressão & $\begin{array}{c}7 \\
(78)\end{array}$ & $\begin{array}{c}7 \\
(78)\end{array}$ & $\begin{array}{c}3 \\
(33)\end{array}$ & $\begin{array}{c}3 \\
(60)\end{array}$ & $\begin{array}{c}4 \\
(80)\end{array}$ & 2 & 0 & 0 & 0 & 0 & 5 \\
\hline QHC - Fator II/Ansiedade & $\begin{array}{c}7 \\
(78)\end{array}$ & $\begin{array}{c}6 \\
(67)\end{array}$ & $\begin{array}{c}6 \\
(67)\end{array}$ & $\begin{array}{c}5 \\
(100)\end{array}$ & $\begin{array}{c}4 \\
(80)\end{array}$ & 0 & 0 & 0 & 0 & 0 & 0 \\
\hline QHC - Fator II/Depressão & 0 & $\begin{array}{c}1 \\
(11)\end{array}$ & $\begin{array}{c}2 \\
(22)\end{array}$ & $\begin{array}{c}2 \\
(40)\end{array}$ & $\begin{array}{c}1 \\
(20)\end{array}$ & 9 & 0 & 0 & 0 & 0 & 0 \\
\hline QHC - Fator III/Ansiedade & $\begin{array}{c}6 \\
(67)\end{array}$ & $\begin{array}{c}7 \\
(78)\end{array}$ & $\begin{array}{c}5 \\
(56)\end{array}$ & $\begin{array}{c}3 \\
(60)\end{array}$ & $\begin{array}{c}5 \\
(100)\end{array}$ & 1 & 1 & 2 & 1 & 0 & 2 \\
\hline QHC - Fator III/Depressão & $\begin{array}{c}3 \\
(33)\end{array}$ & $\begin{array}{c}7 \\
(78)\end{array}$ & $\begin{array}{c}5 \\
(56)\end{array}$ & $\begin{array}{c}3 \\
(60)\end{array}$ & $\begin{array}{c}5 \\
(100)\end{array}$ & 1 & 1 & 2 & 0 & 0 & 0 \\
\hline $\begin{array}{l}\text { QHC - } \\
\text { Potencialidades/Ansiedade }\end{array}$ & $\begin{array}{c}3 \\
(33)\end{array}$ & $\begin{array}{c}3 \\
(33)\end{array}$ & $\begin{array}{c}2 \\
(22)\end{array}$ & $\begin{array}{c}4 \\
(80)\end{array}$ & $\begin{array}{c}2 \\
(40)\end{array}$ & 0 & 7 & 0 & 1 & 1 & 2 \\
\hline $\begin{array}{l}\text { QHC - } \\
\text { Potencialidades/Depressão }\end{array}$ & $\begin{array}{c}3 \\
(33)\end{array}$ & $\begin{array}{c}3 \\
(33)\end{array}$ & $\begin{array}{c}1 \\
(11)\end{array}$ & $\begin{array}{c}4 \\
(80)\end{array}$ & $\begin{array}{c}2 \\
(40)\end{array}$ & 1 & 7 & 0 & 1 & 1 & 2 \\
\hline $\begin{array}{l}\text { QHC - } \\
\text { Dificuldades/Ansiedade }\end{array}$ & $\begin{array}{c}7 \\
(78)\end{array}$ & $\begin{array}{c}5 \\
(56)\end{array}$ & $\begin{array}{c}4 \\
(44)\end{array}$ & $\begin{array}{c}4 \\
(80)\end{array}$ & $\begin{array}{c}3 \\
(60)\end{array}$ & 4 & 3 & 0 & 3 & 0 & 1 \\
\hline $\begin{array}{l}\text { QHC - } \\
\text { Dificuldades/Depressão }\end{array}$ & $\begin{array}{c}7 \\
(78)\end{array}$ & $\begin{array}{c}5 \\
(56)\end{array}$ & $\begin{array}{c}6 \\
(67)\end{array}$ & $3(60)$ & $\begin{array}{c}3 \\
(60)\end{array}$ & 4 & 1 & 0 & 4 & 0 & 1 \\
\hline IHS - Fator Total & $\begin{array}{c}3 \\
(33)\end{array}$ & 0 & $\begin{array}{c}1 \\
(11)\end{array}$ & $\begin{array}{c}1 \\
(20)\end{array}$ & $\begin{array}{c}2 \\
(40)\end{array}$ & 9 & 3 & 3 & 1 & 0 & 0 \\
\hline IHS - Fator I & $\begin{array}{c}5 \\
(56)\end{array}$ & $\begin{array}{c}2 \\
(22)\end{array}$ & $\begin{array}{c}3 \\
(33)\end{array}$ & $\begin{array}{c}3 \\
(60)\end{array}$ & $\begin{array}{c}2 \\
(40)\end{array}$ & 9 & 3 & 0 & 5 & 0 & 0 \\
\hline IHS - Fator II & $\begin{array}{c}3 \\
(33)\end{array}$ & $\begin{array}{c}2 \\
(22)\end{array}$ & $\begin{array}{c}1 \\
(11)\end{array}$ & $\begin{array}{c}2 \\
(40)\end{array}$ & $\begin{array}{c}1 \\
(20)\end{array}$ & 9 & 0 & 0 & 5 & 0 & 0 \\
\hline IHS - Fator III & $\begin{array}{c}2 \\
(22)\end{array}$ & 0 & 0 & $\begin{array}{c}2 \\
(40)\end{array}$ & $\begin{array}{c}2 \\
(40)\end{array}$ & 9 & 3 & 0 & 3 & 1 & 0 \\
\hline IHS - Fator IV & $\begin{array}{c}1 \\
(11)\end{array}$ & 0 & $\begin{array}{c}1 \\
(11)\end{array}$ & $\begin{array}{c}2 \\
(40)\end{array}$ & $\begin{array}{c}2 \\
(40)\end{array}$ & 9 & 1 & 0 & 5 & 0 & 0 \\
\hline IHS - Fator V & $\begin{array}{c}1 \\
(11)\end{array}$ & 0 & $\begin{array}{c}1 \\
(11)\end{array}$ & $\begin{array}{c}1 \\
(20)\end{array}$ & 0 & 8 & 1 & 1 & 5 & 0 & 1 \\
\hline BAl & $\begin{array}{c}1 \\
(11)\end{array}$ & $\begin{array}{c}2 \\
(22)\end{array}$ & 0 & $\begin{array}{c}1 \\
(20)\end{array}$ & $\begin{array}{c}1 \\
(20)\end{array}$ & 6 & 2 & 2 & 4 & 0 & 0 \\
\hline BDI & $\begin{array}{c}1 \\
(11)\end{array}$ & 0 & $\begin{array}{c}1 \\
(11)\end{array}$ & 0 & 0 & 8 & 4 & 0 & 5 & 0 & 0 \\
\hline Mini-SPIN & $\begin{array}{c}5 \\
(56)\end{array}$ & $\begin{array}{c}1 \\
(11)\end{array}$ & $\begin{array}{c}1 \\
(11)\end{array}$ & $\begin{array}{c}2 \\
(40)\end{array}$ & $\begin{array}{c}2 \\
(40)\end{array}$ & 4 & 4 & 0 & 5 & 0 & 0 \\
\hline
\end{tabular}




\section{W'INTERACÃO EM ET PSICOLOGIA}

Partindo do número de participantes clínicos, descritos na Tabela 2, verifica-se que os grupos tinham um repertório de entrada equivalente. Desse modo, as mudanças entre as avaliações podem ser atribuídas à intervenção realizada. Considerando os indicadores clínicos de ansiedade e depressão pelos instrumentos BAI e BDI constatou-se que os universitários, em sua grande maioria, não apresentavam tais indicadores clínicos, por isso, pode-se afirmar que a amostra era composta especialmente por pessoas não clínicas para ansiedade e depressão. No entanto, a maioria dos participantes (cinco de nove) apresentaram indicadores de fobia social pela Mini-SPIN, que é um instrumento rastreador e, após a intervenção, apenas um desses participantes ainda mantinha o indicador, os demais, não. Tanto nas medidas do QHC como no IHS-Del Prette, verificou-se um maior número de aquisições comportamentais no Grupo Experimental que no Grupo Controle.

Tendo por parâmetro o Índice de Significância Clínica (ISC) dos fatores dos instrumentos, verificou-se que os participantes, em ambos os grupos, já eram considerados população funcional para a grande parte dos indicadores:
$56 \%$ do Grupo Experimental e 51\% do Grupo Controle apresentavam, já no pré-teste, um ISC compatível com a população funcional, isto é, sem problemas de saúde mental. Notou-se entretanto que, no Grupo Experimental, houve um número maior de mudanças funcionais (24\%), indicativas de melhora decorrente da intervenção, as quais ocorreram em treze indicadores, considerando os instrumentos de habilidades sociais e saúde mental. Mudanças disfuncionais (MD) aconteceram em apenas cinco indicadores desses mesmos instrumentos, que representam $6 \%$ das possíveis alterações nos fatores estudados. Já o Grupo Controle apresentou mudanças funcionais (MF) em apenas dois indicadores do QHC e um do IHS, correspondendo a 3\% de mudanças, enquanto as mudanças disfuncionais (MD) ocorreram em sete indicadores, considerando o QHC e o IHS, num total em $14 \%$ das alterações. Conclui-se, ao verificar os totais ponderados, que as mudanças funcionais (MF) foram muito mais frequentes no Grupo Experimental e as mudanças disfuncionais (MD) ocorreram mais no Grupo Controle. A Tabela 3 descreve os resultados das comparações estatísticas entre as medidas de avaliação nos dois grupos.

Tabela 3. Teste de Wilcoxon referente aos indicadores avaliados, considerando os escores brutos

\begin{tabular}{|c|c|c|c|c|c|c|c|c|c|}
\hline \multicolumn{10}{|c|}{ Análise estatística das competências avaliadas } \\
\hline \multirow{3}{*}{ Indicadores } & \multicolumn{6}{|c|}{ Grupo Experimental } & \multicolumn{3}{|c|}{ Grupo Controle } \\
\hline & \multicolumn{3}{|c|}{ Mediana } & \multicolumn{3}{|c|}{ Valor de $p$} & \multicolumn{2}{|c|}{ Mediana } & \multirow{2}{*}{$\begin{array}{l}\text { Valor de } p \\
\text { LB1/LB2 }\end{array}$} \\
\hline & Pré & Pós & Seg & Pré/Pós & Pré/Seg & Pós/Seg & LB1 & LB2 & \\
\hline QHC - Fator 1 & 15 & 17 & 16 & 0,397 & 0,120 & 0,674 & 18 & 18 & 0,29 \\
\hline QHC - Fator 2 & 4 & 6 & 6 & 0,135 & 0,061 & 0,121 & 4 & 4 & 0,22 \\
\hline QHC - Fator 3 & 4 & 4 & 25 & 0,609 & 0,905 & 0,480 & 5 & 4 & 0,19 \\
\hline $\begin{array}{l}\text { QHC - } \\
\text { Situação/Assunto } \\
\text { QHC - }\end{array}$ & 27 & 32 & 34 & 0,674 & 0,270 & 0,063 & 23 & 24 & 0,89 \\
\hline $\begin{array}{l}\text { Comportamento } \\
\text { Habilidoso }\end{array}$ & 13 & 32 & 14 & 0,008 & 0,671 & 0,008 & 10 & 10 & 0,72 \\
\hline $\mathrm{QHC}-$ & & & & & & & & & \\
\hline $\begin{array}{l}\text { Comportamento Não } \\
\text { Habilidoso }\end{array}$ & 3 & 0 & 1 & 0,041 & 0,495 & 0,168 & 1 & 1 & 0,71 \\
\hline $\begin{array}{l}\text { QHC - Consequência } \\
\text { Positiva }\end{array}$ & 27 & 25 & 31 & 0,767 & 0,056 & 0,352 & 20 & 24 & 0,34 \\
\hline $\begin{array}{l}\text { QHC - Consequência } \\
\text { Negativa }\end{array}$ & 4 & 2 & 3 & 0,394 & 0,726 & 0,891 & 2 & 2 & 0,41 \\
\hline $\begin{array}{l}\text { QHC - Sentimento } \\
\text { Positivo }\end{array}$ & 4 & 38 & 45 & 0,008 & 0,008 & 0,069 & 21 & 25 & 0,89 \\
\hline $\begin{array}{l}\text { QHC - Sentimento } \\
\text { Negativo }\end{array}$ & 18 & 10 & 9 & 0,044 & 0,192 & 0,395 & 16 & 17 & 0,89 \\
\hline $\begin{array}{l}\text { QHC - } \\
\text { Potencialidades }\end{array}$ & 60 & 107 & 124 & 0,858 & 0,008 & 0,017 & 66 & 80 & 0,27 \\
\hline QHC - Dificuldades & 21 & 21 & 20 & 0,240 & 0,155 & 0,182 & 20 & 19 & 0,89 \\
\hline IHS - Total & 30 & 65 & 55 & 0,011 & 0,011 & 1,000 & 35 & 65 & 0,27 \\
\hline IHS - Fator I & 25 & 65 & 70 & 0,012 & 0,018 & 0,236 & 15 & 70 & 0,10 \\
\hline IHS - Fator II & 25 & 35 & 45 & 0,350 & 0,017 & 0,057 & 35 & 50 & 0,36 \\
\hline IHS - Fator III & 45 & 70 & 80 & 0,008 & 0,012 & 0,574 & 30 & 45 & 0,50 \\
\hline IHS - Fator IV & 45 & 65 & 55 & 0,021 & 0,137 & 0,157 & 35 & 25 & 0,68 \\
\hline IHS - Fator V & 75 & 75 & 60 & 0,799 & 0,351 & 0,043 & 85 & 40 & 0,22 \\
\hline $\mathrm{BAI}$ & 4 & 3 & 7 & 0,953 & 0,373 & 0,623 & 15 & 12 & 0,46 \\
\hline BDI & 6 & 4 & 4 & 0,017 & 0,068 & 0,248 & 9 & 11 & 0,85 \\
\hline Mini-SPIN & 6 & 2 & 4 & 0,007 & 0,048 & 0,047 & 3 & 3 & 0,10 \\
\hline
\end{tabular}




\section{MI* INTERACÃO EM ET. PSICOLOGIA}

De acordo com a Tabela 3, no que se refere ao IHS-Del Prette, nota-se que no Grupo Experimental houve mudança significativa em todos os fatores avaliados, cujo repertório aumentou após a intervenção e se manteve na avaliação de seguimento de seis meses. A análise estatística do QHCUniversitários constatou mudanças significativas de repertório entre pré e pós-teste, pré-teste e seguimento. e entre pós-teste e seguimento. Em relação ao BDI, a melhora foi significativa entre as avaliações de pré e pós-teste. $\mathrm{Na}$ Mini-SPIN também foi verificada melhora entre os escores de pré e pós-teste, pré-teste e seguimento, e pós-teste e seguimento. Já para o Grupo Controle, a análise estatística não apontou significância em nenhum dos indicadores nas avaliações.

\section{DISCUSSÃO}

De maneira geral o procedimento de intervenção Promove-Universitários atestou eficácia nas comparações entre GE e GC. As queixas relatadas pelos participantes corresponderam ao já elucidado pela literatura (Bolsoni-Silva \& Loureiro, 2017; Leme et al., 2017; Ribeiro \& Bolsoni-Silva, 2011), confirmando que as motivações, de maneira geral, para buscar atendimento foram: falar em público, dificuldade de relacionamento interpessoal (namoros, familiares e amigos), autocontrole da agressividade e autoconhecimento. É importante destacar que o procedimento, ainda que semiestruturado, de acordo com a prática baseada em evidências (APA, 2006), ensina comportamentos identificados como relevantes a essa população de acordo com as diferentes demandas. Como a análise funcional é o foco da intervenção (Abreu \& Abreu, 2017), ela possivelmente garantiu motivação (Goldiamond, 2002) para engajar-se no tratamento, por um lado, e, por outro, favoreceu autoconhecimento quanto às dificuldades enfrentadas nas interações sociais.

De maneira geral os resultados demonstraram que o Promove-Universitários diminuiu indicadores de fobia social, ansiedade e depressão, mesmo tendo poucos indicadores no pré-teste. Desse modo, atestou-se que o ensino de habilidades sociais na terapia analítico-comportamental foi suficiente para promover saúde mental, de forma semelhante ao verificado em outras intervenções (Ferreira et al., 2014; Klein et al., 2011; Orti et al., 2015; Probst et al., 2017; Räsänen et al., 2016; Rocha et al., 2012), o que pode melhorar o desempenho acadêmico (Brandão et al., 2017) e diminuir o risco de suicídio (Tang et al., 2018).

As mudanças foram, em maior número, funcionais no Grupo Experimental e, em maior número, disfuncionais no Grupo Controle, o que reafirma a validade interna do procedimento (Z. A. P. Del Prette \& Del Prette, 2008), semelhante ao encontrado por Lima e Soares (2015) e atestando que o aprendizado é mais eficiente com o ensino programado (Murta, 2005). O estudo de Lopes et al. (2017), também utilizou o método JT (Z. A. P. Del Prette \& Del Prette, 2008), encontrando resultados semelhantes ao deste estudo tanto em mudanças disfuncionais (MD) quanto em funcionais (MF).

Desse modo, pode-se concluir que o PromoveUniversitário foi eficaz para reduzir sintomas de ansiedade, depressão e fobia social (Ferreira et al., 2014; Orti et al., 2015; Rocha et al., 2012) avançando metodologicamente, pelo delineamento experimental de grupo. 0 presente estudo testou o programa em grupo que permite a aplicação em maior número de pessoas, mantendo os resultados obtidos com atendimento individual.

O presente estudo também demonstrou que a aquisição de habilidades sociais reduz problemas interpessoais, tal como já verificado na literatura (Bolsoni-Silva, et al., 2009; Ferreira et al., 2014; Orti et al., 2015; Lima \& Soares, 2015; Lopes et al., 2017; Rocha et al., 2012). Os achados desta pesquisa reafirmam o caráter de promoção de habilidades sociais e de prevenção de déficits interpessoais (Abreu et al., 2016), isto é, programas de promoção de habilidades sociais poderiam ser conduzidos antes mesmo de os universitários terem cronicidade em problemas acadêmicos e de saúde mental (Brandão, 2016; Brandão et al., 2017), já que o ingresso na universidade não garante a permanência e formatura (INEP, 2017).

Como afirmaram Segrin e Flora (2000), baixo repertório de habilidades sociais dificulta o enfrentamento de situações estressantes que podem ser gatilhos para transtornos psicológicos. Adicionalmente, Goldiamond (2002) já anunciava que o repertório de habilidades sociais poderia ser um comportamento funcionalmente equivalente, capaz de aumentar a probabilidade de a pessoa obter reforçadores. Com os dados da presente pesquisa, concorda-se que habilidades sociais sejam operantes (Bolsoni-Silva, 2002) que podem funcionar como funcionalmente equivalentes aos sintomas apresentados (Bolsoni-Silva \& Carrara, 2010).

Os achados deste estudo alertam para a necessidade de políticas públicas junto às universidades de forma a garantir que os estudantes tenham acesso a informação e atendimento psicológico, de maneira preventiva (Abreu et al., 2016), de forma a favorecer repertórios e saúde necessários à sua permanência na universidade. 


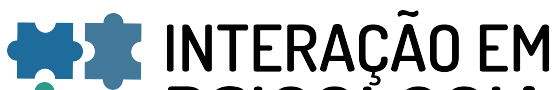 ET PSICOLOGIA}

\section{CONSIDERAÇÕES FINAIS}

O presente estudo verificou a eficácia do PromoveUniversitários, aplicado em grupo, seja na aquisição de repertórios, seja na redução de dificuldades, queixas e problemas de saúde mental.

Como pontos fortes da investigação destacam-se: a) uso de múltiplos instrumentos para avaliar dificuldades e potencialidades nas interações sociais, bem como indicadores de saúde mental; b) o uso de diversos métodos de análise de dados (qualitativo com a entrevista clínica, método JT e estatístico); c) uso do controle experimental com distribuição aleatória entre os grupos; e d) uso de medidas de seguimento para verificar a manutenção dos resultados.

No entanto, a amostra do estudo é bem reduzida e inclui estudantes de universidades pública e privada, que são variáveis que podem ter interferido nos resultados e devem ser controladas. Tais limitações não permitiram análises estatísticas mais robustas, com uso de estatística paramétrica, bem como não permitem fazer generalização dos resultados.

Estudos futuros com o programa podem, então, ampliar a amostra, homogeneizar a população e incluir medidas de observação. É importante que o Promove-Universitário seja amplamente aplicado e avaliado em diferentes estados do Brasil, incluindo universidades públicas e privadas, nas modalidades individual e grupal, na prevenção, na remediação e na promoção de saúde, de forma a avaliar sua validade externa.

\section{DECLARAÇÃO DE FINANCIAMENTO}

A pesquisa relatada no manuscrito foi financiada parcialmente pela bolsa de mestrado da primeira autora (CNPq)

\section{DECLARAÇÃO DA CONTRIBUIÇÃO DOS AUTORES}

L. A. M.: coleta de dados, análise de dados e revisão de literatura.

A. T. B.: orientação e revisão final do texto.

\section{DECLARAÇÃO DE CONFLITOS DE INTERESSE}

Os autores declaram que não há conflitos de interesse no manuscrito submetido.

\section{REFERÊNCIAS}

Abreu, P., \& Abreu, J. (2017). A quarta geração de terapias comportamentais. Revista Brasileira de Terapia Comportamental e Cognitiva, 19(3), 190-211. doi:10.31505/ rbtcc.v19i3.1069

Abreu, S., Miranda, A. A. V., \& Murta, S. G. (2016). Programas preventivos brasileiros: quem faz e como é feita a prevenção em saúde mental? Psico-USF, 21(1), 163-177. doi:10.1590/1413-82712016210114.

Beck, A. T., Epstein, N., Brown, G., \& Steer, R. A. (1988). An inventory for measuring clinical anxiety: Psychometric properties. Journal of Consulting and Clinical Psychology, 56(6), 893-897. doi:10.1037/0022-006X.56.6.893

Beck, A. T. \& Steer, R. A. (1993). Beck Depression Inventory. Manual. San Antonio: Psychology Corporation.

Beidel, D. C., Alfano, C. A., Kofler, M. J., Rao, P. A., Scharfstein, L., Sarver, N. W. (2014). The impact of social skills training for social anxiety disorder: A randomized controlled trial. Journal of Anxiety Disorders, 28(8), 908-918. doi:10.1016/ j.janxdis.2014.09.016.

Bolsoni-Silva, A. T. (2002). Habilidades sociais: breve análise da teoria e da prática à luz da análise do comportamento. Interação em Psicologia, 6(2), 233-242. doi:10.5380/ psi.v6i2.3311.

Bolsoni-Silva, A. T. (2009a). Como enfrentar os desafios da universidade. São Carlos: Suprema.

Bolsoni-Silva, A. T. (2009b). Habilidades sociais de universitários: Procedimentos de intervenção na perspectiva da análise do comportamento. In R. C. Wielenska (Ed.), Sobre comportamento e cognição desafios, soluções e questionamentos (Vol. 23, pp. 21-52). Santo André: ESETec.

Bolsoni-Silva, A. T., \& Carrara, K. (2010). Habilidades sociais e análise do comportamento: compatibilidades e dissensões conceitual-metodológicas. Psicologia em Revista, 16(2), 330-350.

Bolsoni-Silva, A. T., Leme, V. B. R., Lima, A. M. A., Costa-Júnior, F. M., \& Correia M. R. G. (2009). Avaliação de um treinamento de habilidades sociais (THS) com universitários e recém-formados. Interação em Psicologia, 13(2), 241-251. doi:10.5380/psi.v13i2.13597.

Bolsoni-Silva, A. T., \& Carrara, K. (2010). Habilidades sociais e análise do comportamento: compatibilidades e dissensões conceitual-metodológicas. Psicologia em Revista, 16(2), 330-350.

Bolsoni-Silva, A. T., \& Loureiro, S. R. (2017). Teste psicológico - Questionário de Avaliação de Habilidades Sociais, Comportamentos e Contextos para Universitários (QHCUNIVERSITÁRIOS). São Paulo: Hogrefe. 


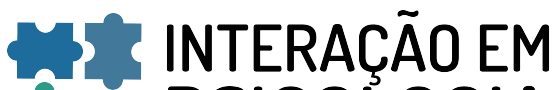 ET PSICOLOGIA}

Brandão, A. S. (2016). Desempenho acadêmico de universitários, variáveis preditoras: habilidades sociais, saúde mental, características demográficas e escolares. (Tese de Doutorado). Faculdade de Filosofia, Ciências e Letras de Ribeirão Preto, Universidade de São Paulo, Ribeirão Preto, SP.

Brandão, A. S., Bolsoni-Silva, A. T., \& Loureiro, S. R. (2017). The Predictors of Graduation: Social Skills, Mental Health, Academic Characteristics. Paidéia, 27(66), 117-125. doi: 10.1590/1982-43272766201714.

Connor, K.M.; Kobak, K.A.; Churchill, L.E.; Katzelnick, D.; Davidson, J.R.T. (2001). Mini-SPIN: abrief screening assessment for generalized social anxiety disorder. Depress Anxiety, 14(2), 137-140.

Cozby, P. C. (2003). Métodos de pesquisa em ciências do comportamento. São Paulo: Atlas.

Cunha, J. A. (2001). Manual da versão em português das Escalas Beck. São Paulo: Casa do Psicólogo.

Del Prette, A., \& Del Prette, Z. A. P. (2001). Psicologia das relações interpessoais: vivências para o trabalho em grupo. Petrópolis: Vozes.

Del Prette, Z. A. P., \& Del Prette, A. (2001). Inventário de habilidades sociais (IHS-Del-Prette): Manual de aplicação, apuração e interpretação. São Paulo: Casa do Psicólogo.

Del Prette, Z. A. P., \& Del Prette, A. (2008). Significância Clínica e Mudança Confiável na Avaliação de Intervenções Psicológicas. Psicologia: Teoria e Pesquisa, 24(4), 105114. doi:10.1590/S0102-37722008000400013.

Ferreira, V. S., Oliveira, M. A., \& Vandenberghe, L. (2014). Efeitos a curto e longo prazo de um grupo de desenvolvimento de habilidades sociais para universitários. Psicologia: Teoria e Pesquisa, 30(1), 73-81. doi:10.1590/S0102-37722014000100009.

Ferster, C. B. (1973). A functional analysis of depression. American Psychologist, 28(10), 857-870. doi:10.1037/ h0035605

Goldiamond, I. (2002). Toward a constructional approach to social problems: Ethical and constitutional issues raised by applied behavioral analysis. Behavior and Social Issues, 11(2), 108-197. doi:10.5210/bsi.v11i2.92.

Instituto Nacional de Estudos e Pesquisas Educacionais Anísio Teixeira (2014). Resumo técnico: censo da educação superior 2014. Brasília, DF: INEP. Recuperado de http://download.inep.gov.br/download/superior/ censo/2014/ resumo_tecnico_censo_educacao_superior_2014.pdf
Klein, D. N., Leon, A. C., Li, C., D'Zurilla, T. J., Black, S. R., Vivian, ... Kocsis, J. H. (2011). Social problem solving and depressive symptoms over time: A randomized clinical trial of cognitive-behavioral analysis system of psychotherapy, brief supportive psychotherapy, and pharmacotherapy. Journal of Consulting and Clinical Psychology, 79 (3), 342-352.

Leme, V. B. R., Del Prette, Z. A., \& Del Prette, A. (2016). Habilidades sociais de estudantes de psicologia: Estado da arte no Brasil. In A. B. Soares, L. Mourão, \& M. M. P. E. Mota (Orgs.), $O$ Estudante Universitário Brasileiro: características cognitivas, habilidades relacionais e transição para o mercado de trabalho (pp.127-142). Curitiba: Appris.

Lima, C. A., \& Soares, A. B. (2015). Treinamento em Habilidades Sociais para universitários no contexto acadêmico: ganhos e potencialidades em situações consideradas difíceis. In Z. A. P. Del Prette, A. B. Soares, C. S. Pereira-Guizzo, M. F. Wagner, \& V. B. R. Leme (Orgs.), Habilidades sociais - diálogos e intercâmbios sobre pesquisa e prática (pp. 22-43). Novo Hamburgo: Sinopsys.

Lopes, D. C., Dascanio, D., Ferreria, B. C., Del Prette, Z. A. P., \& Del Prette, A. (2017). Treinamento de habilidades sociais: Avaliação de um programa de desenvolvimento interpessoal profissional para universitários de ciências exatas. Interação em Psicologia, 21(1), 55-65. doi:10.5380/ psi.v21i1.36210.

Meyer, S. B., Del Prette, G., Zamignani, D. R., Banaco, R. A., Neno, S., \& Tourinho, E. Z. (2010) Análise do comportamento e terapia analítico-comportamental. In: E. Z. Tourinho \& S. V. Luna (Orgs.), Análise do comportamento: Investigações históricas, conceituais e aplicadas (153-174). São Paulo: Roca.

Orti, N. P., Souza-Girotti, V. B., \& Bolsoni-Silva, A. T. (2015). Social skills training in behavior therapy with college students diagnosed with depression. International Journal of Current Research, 7(4), 15382-15391.

Osório, F. L., Crippa, J. A. S., \& Loureiro, S. R. (2007). A study of the discriminative validity of a screening tool (MINISPIN). European Psychiatry, 22(4), 239-249. doi:10.1016/ j.eurpsy.2007.01.003.

Probst, T., Geib, C., Güroff , E., \& Mühlberger, A. (2017). Training the social skill "being able to demand" vs. training the social skill "being able to say no". A randomized controlled trial with healthy individuals. Journal of Behavior Therapy and Experimental Psychiatry, 57, 1-5. doi: 10.1016/j.jbtep.2017.01.006 


\section{-4: INTERACÃO EM LF PSICOLOGIA}

Räsänen, P., Lappalainen, P., Muotka, J., Tolvanen, A., \& Lappalainen, R. (2016). An online guided ACT intervention for enhancing the psychological wellbeing of university students: A randomized controlled clinical trial. Behaviour Research and Therapy, 78, 30-42. doi:10.1016/j.brat. 2016.01.001

Ribeiro, D., \& Bolsoni-Silva, A. T. (2011). Potencialidades e dificuldades interpessoais de universitários: estudo de caracterização. Acta Comportamentalia, 19(2), 205-224.

Rocha, J. F., Bolsoni-Silva, A. T., \& Verdu, A. C. M. A. (2012). O uso do treino de habilidades sociais em pessoas com fobia social na terapia comportamental. Perspectivas em Análise do Comportamento, 3(1), 38-56.

Segrin, C., \& Flora, J. (2000). Poor social skills are a vulnerability factor in the development of psychosocial problems. Human Communication Research, 26(3), 489514. doi:10.1111/j.1468-2958.2000.tb00766.x.

\section{Laisa Aparecida Moretto e Alessandra Turini Bolsoni-Silva}

Skinner, B. F (1974). Sobre o Behaviorismo. São Paulo: Ed. Cultrix.

Tang, F., Byrne, M., \& Qin, P. (2018). Psychological distress and risk for suicidal behavior among university students in contemporary China. Journal of Affective Disorders, 228, 101-108. doi:10.1016/j.jad.2017.12.005.

Zamignani, D., \& Banaco, R. (2005). Um Panorama AnalíticoComportamental sobre os Transtornos de Ansiedade. Revista Brasileira de Terapia Comportamental e Cognitiva, 7(1), 77-92. doi:10.31505/rbtcc.v7i1.44

Data de submissão: 22/08/2018 Primeira decisão editorial: 02/10/2018 Aceite em 22/11/2018 